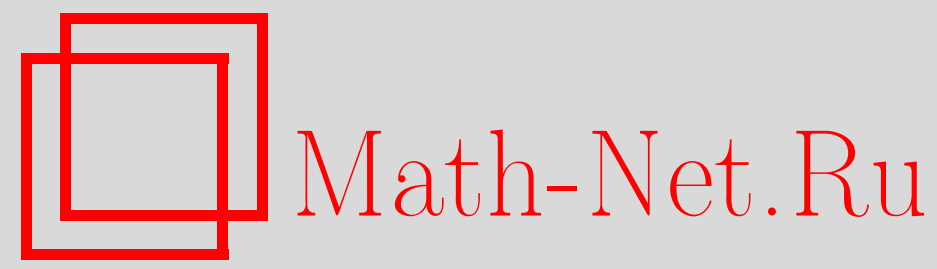

Б. И. Селиванов, Об одном обобщении классической задачи о дробинках, Теория вероятн. и ее примен., 1998, том 43, выпуск 2, 315-330

DOI: https://doi.org/10.4213/tvp1467

Использование Общероссийского математического портала Math-Net.Ru подразумевает, что вы прочитали и согласны с пользовательским соглашением http://www.mathnet.ru/rus/agreement

Параметры загрузки:

IP : 54.162 .27 .143

26 апреля 2023 г., 15:30:28

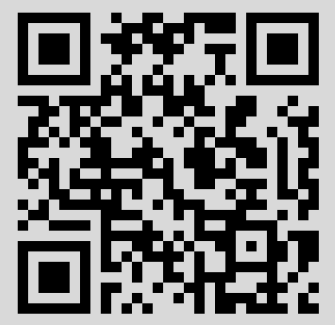


(C) 1998 г.

\author{
СЕЛИВАНОВ Б. И.
}

\title{
ОБ ОДНОМ ОБОБЩЕНИИ КЛАССИЧЕСКОЙ ЗАДАЧИ О ДРОБИНКАХ ${ }^{1)}$
}

В работе несколько известных предельных теорем для классической задачи о дробинках, включая локальную предельную теорему, перенесены на одно семейство условных распределений, связанных со случайными равновероятными размешениями частиц в ячейках двумерных таблиц.

Ключевые слова и фразы: случайные размещения, классическая задача о дробинках, условное распределение, двумерная таблица, закон Пуассона, нормальное распределение, локальная предельная теорема, метод перевала.

1. Введение. Классическая задача о дробинках $[1$, гл. 1] обобщалась многими авторами в различных направлениях. В настоящей работе, опирающейся на некоторые результаты предыдущей статьи автора [2], намечается еще одно направление обобщений этой задачи.

Пусть дана двумерная таблица $T$, имеющая $N$ строк и $M$ столбцов, $N, M \geqslant 1$. В ее $N M$ клетках (ячейках) случайно независимо друг от друга размешаются частищы (дробинки) так, что вероятность попадания каждой частицы в любую из клеток таблицы $T$ равна $(N M)^{-1}$. Назовем строку таблицы $T$ пустой, если все $M$ ее клеток пусты, и непустой в противном случае. Введем для каждого $n=1,2, \ldots$ следующие события, связанные с размещением $n$ частищ в таблице $T$ :

$\mathscr{A}_{n}$ - «в каждой строке таблицы $T$ заполнено не более одной клетКи»,

$\mathscr{B}_{n k}-\ll$ в $T$ имеется ровно $k$ пустых строк»,

$\mathscr{C}_{n k}-\ll$ в $T$ имеется ровно $k$ непустых строк», $k=0,1, \ldots, N$. Положим $\pi_{n}=\pi_{n}(N, M)=\mathbf{P}\left\{\mathscr{A}_{n}\right\}, \tau_{n k}=\tau_{n k}(N$, $M)=\mathbf{P}\left\{\mathscr{B}_{n k} \mathscr{A}_{n}\right\}$ и $\pi_{n k}=\pi_{n k}(N, M)=\mathbf{P}\left\{\mathscr{C}_{n k} \mathscr{A}_{n}\right\}, n=1,2, \ldots, k=$ $0,1, \ldots, N$, и рассмотрим условные вероятности

$$
\widehat{\tau}_{n k}=\widehat{\tau}_{n k}(N, M)=\mathbf{P}\left\{\mathscr{B}_{n k} \mid \mathscr{A}_{n}\right\}=\frac{\tau_{n k}}{\pi_{n}} .
$$

*Работа выполнена при финансовой поддержке Российского фонда фундаментальных исследований (проект № 96-01-00531).

1) Математический институт им. В. А. Стеклова РАН, ул. Губкина, 8, 117966 Москва, ГСІІ-1, Россия. 
Для каждого $n=1,2, \ldots$ вероятности (1.1) определяют распределение некоторой случайной величины (сл.в.) $\widehat{\mu}_{0}=\widehat{\mu}_{0}(n, N, M)$, для которой $\mathbf{P}\left\{\widehat{\mu}_{0}=k\right\}=\widehat{\tau}_{n k}, k=0,1, \ldots, N$. Если $M=1$, то $\pi_{n}=1$ для всех $n=1,2, \ldots$ и сл.в. $\hat{\mu}_{0}$ есть число $\mu_{0}=\mu_{0}(n, M)$ пустых ячеек в классической задаче о дробинках. В обшем случае при $M \geqslant 2$ сл.в. $\widehat{\mu}_{0}$ можно интерпретировать как число пустых строк в таблице $T$ после размещения в ней $n$ частищ при условии, что все непустые клетки находятся в различных строках.

В настоящей работе, предполагая, что $n, N \rightarrow \infty$, а $M \geqslant 2$ фиксированно, на семейство сл.в. $\widehat{\mu}_{0}(n, N, M), M=2,3, \ldots$, перенесены несколько известных предельных теорем для сл.в. $\mu_{0}(n, M)$. В п. 2 в левой области изменения параметров $n$ и $N$

$$
\frac{n^{2}}{2 N} \rightarrow \lambda, \quad n, N \rightarrow \infty, 0<\lambda<\infty, \lambda \text { - константа, }
$$

доказана сходимость по распределению сл.в. $\widehat{\mu}_{0}(n, N, M)-N+n$ к пуассоновской сл.в. с параметром $\lambda / M$. Соответствующее утверждение для сл.в. $\mu_{0}(n, N)$, различные доказательства которого можно найти в [1] и [3], впервые было указано в работе [4].

В п. 3 доказываются некоторые вспомогательные предложения. В частности, в центральной области изменения параметров $n$ и $N$

$$
\frac{n}{N}=\alpha, \quad n, N \rightarrow \infty, 0<\alpha_{0} \leqslant \alpha \leqslant \alpha_{1}<\infty, \alpha_{0}, \alpha_{1} \text { - константы, }
$$

получены асимптотические оценки для вероятностей $\pi_{n}(N, M)$ и величин $\mathbf{E} \widehat{\mu}_{0}(n, N, M), \mathbf{D} \widehat{\mu}_{0}(n, N, M)$.

В п. 4 доказан основной результат статьи - локальная предельная теорема для сл.в. $\widehat{\mu}_{0}(n, N, M)$. При этом предполагается, что $M \geqslant 2-$ фиксированное число и выполняется условие (1.3). Доказательство теоремы проводится с использованием метода перевала по схеме, аналогичной разработанной Севастьяновым Б. А. и Чистяковым В. П. в [5]. Дополнительные технические трудности связаны с присутствием во всех рассуждениях корня $x_{0}$ уравнения (3.3) (см. ниже). В заключение п. 4 для сл.в. $\widehat{\mu}_{0}(n, N, M)$ формулируется центральная.предельная теорема; справедливость ее установлена при тех же условиях, при которых выполняется локальная предельная теорема.

Результаты работы могут быть использованы, например, при рассмотрении системы случайных уравнений вида

$$
x_{\varepsilon_{i}}=\eta_{i}, \quad i=1, \ldots, n,
$$

c $N$ неизвестными $x_{1}, \ldots, x_{N}$, принимающими значения $1, \ldots, M$. Предположим, что индексы неизвестных $\varepsilon_{i}$ и правые части $\eta_{i}$ уравнений (1.4) - независимые сл.в. и $\varepsilon_{i}$ и $\eta_{i}$ для каждого $i=1, \ldots, n$ с равными вероятностями принимают значения $1, \ldots, N$ и $1, \ldots, M$ соответственно. 
Тогда $\pi_{n}$ есть вероятность совместности системы (1.4), а распределение числа неизвестных, неопределенных из системы (1.4), при условии, что система совместна, задается вероятностями (1.1) и, следовательно, совпадает с распределением сл.в. $\widehat{\mu}_{0}$.

Автор благодарен А. М. Зубкову, замечания которого позволили улучшить изложение материалов настоящей работы.

2. Согласно [2] для производящей функции (ІІ.ф.) вероятностей $\pi_{n k}(N, M)$

$$
P_{N M}(x, z)=1+\sum_{n=1}^{\infty} \sum_{k=0}^{N} \pi_{n k} x^{k} \frac{z^{n}}{n !}
$$

выполняется соотношение

$$
P_{N M}(x, N M z)=\left(1+M x\left(e^{z}-1\right)\right)^{N} .
$$

Разлагая в ряд по степеням $x$ и $z$ правую часть (2.2), найдем

$$
\pi_{n k}=\frac{1}{N^{n} M^{n-k}}\left(\begin{array}{c}
N \\
k
\end{array}\right) \sum_{m=1}^{k}(-1)^{k-m}\left(\begin{array}{c}
k \\
m
\end{array}\right) m^{n}
$$

где $n=1,2, \ldots, k=1, \ldots, N$, (ясно, что $\left.\pi_{n 0}=0\right)$. Формула (2.3) позволяет находить также вероятности $\tau_{n k}$, так как

$$
\tau_{n k}=\pi_{n, N-k}, \quad n=1,2, \ldots, k=0,1, \ldots, N .
$$

По смыслу вероятностей $\pi_{n}$ и $\pi_{n k}$

$$
\pi_{n}=\sum_{k=0}^{N} \pi_{n k}, \quad n=1,2, \ldots
$$

Отсюда и из (2.3) следует, что $\pi_{n}=1$, если $M=1$, и

$$
\pi_{n}=\frac{(M-1)^{N}}{N^{n} M^{n}} \sum_{m=1}^{N}(-1)^{N-m}\left(\begin{array}{l}
N \\
m
\end{array}\right)\left(\frac{M}{M-1}\right)^{m} m^{n}
$$

где $n=1,2, \ldots, M=2,3 \ldots$.

Введем п.ф. вероятностей $\tau_{n k}$ :

$$
T_{N, M}(x, z)=x^{N}+\sum_{n=1}^{\infty} \sum_{k=0}^{N} \tau_{n k} x^{k} \frac{z^{n}}{n !}
$$

Из равенства (2.4) и представления (2.2) п.ф. (2.1) следует, что

$$
T_{N, M}(x, z)=\left(x+M\left(e^{z /(N M)}-1\right)\right)^{N} .
$$


С помощью формул $(1.1),(2.5)$ и (2.6) найдем факториальные моменты $\mathrm{E} \widehat{\mu}_{0}^{[l]}$ сл.в. $\widehat{\mu}_{0}(n, N, M)$ (воспользуемся обозначением $a^{[l]}=a(a-1) \cdots$ $(a-l+1))$ :

$$
\begin{aligned}
\mathbf{E} & \widehat{\mu}_{0}^{[l]}(n, N, M)=\left.\frac{n !}{\pi_{n}} \operatorname{Coeff}_{z^{n}} \frac{\partial^{l}}{\partial x^{l}} T_{N, M}(x, z)\right|_{x=1} \\
= & \frac{n !}{\pi_{n}} N^{[l]} \operatorname{Coeff}_{z^{n}}\left(1+M\left(e^{z /(N M)}-1\right)\right)^{N-l}, \quad n, l=1,2, \ldots .
\end{aligned}
$$

IІри $M=1$ равенство (2.7) принимает вид

$$
\mathbf{E} \widehat{\mu}_{0}^{[l]}(n, N)=N^{[l]}\left(1-\frac{l}{N}\right)^{n}
$$

(см., например, $[1$, с. 17]). Если же $M \geqslant 2$, то

$$
\begin{aligned}
\mathbf{E} \hat{\mu}_{0}^{[l]}(n, N, M)= & \frac{N^{[l]}}{\pi_{n} N^{n} M^{n}}(M-1)^{N-l} \\
& \times \sum_{m=1}^{N-l}(-1)^{N-l-m}\left(\begin{array}{c}
N-l \\
m
\end{array}\right)\left(\frac{M}{M-1}\right)^{m} m^{n},
\end{aligned}
$$

где $n, l=1,2, \ldots$. В частности, при $l=1$ из (2.8) и (2.9) получаем выражение для математического ожидания $\widehat{\mu}_{0}(n, N, M):$ при $n=1,2, \ldots$

$$
\mathbf{E} \widehat{\mu}_{0}(n, N, M)= \begin{cases}N\left(1-\frac{1}{N}\right)^{n}, & \text { если } M=1, \\
\frac{(M-1)^{N-1}}{\pi_{n}(N, M) N^{n-1} M^{n}} & \\
\times \sum_{m=1}^{N-l}(-1)^{N-m-1}\left(\begin{array}{c}
N-1 \\
m
\end{array}\right)\left(\frac{M}{M-1}\right)^{m} m^{n}, \\
\text { если } M=2,3, \ldots .\end{cases}
$$

Теорема 2.1. Если при $n \rightarrow \infty$ иисло строк $N$ табличы $T$ изменяется так, что выполняется условие (1.2), а число ее столбиов $M \geqslant 2$ остается фиксированньм, то

$$
\widehat{\mu}_{0}(n, N, M)-N+n \underset{N \rightarrow \infty}{\Longrightarrow} \prod\left(\frac{\lambda}{M}\right)
$$

где $\mathrm{I}(a)-c л . в .$, распределенная по закону ІІассона с параметром а, и символ $\Longrightarrow$ означает сходимость сл.в. по распределению. 
Д ок а з а т е л ь с т в о. Учитывая равенства $(2.3),(2.4)$ и используя известную формулу

$$
\begin{aligned}
& \mathbf{P}\left\{\mu_{0}(n, N)=k\right\} \\
& \quad=\left(\begin{array}{c}
N \\
k
\end{array}\right) \sum_{m=0}^{N-k}(-1)^{m}\left(\begin{array}{c}
N-k \\
m
\end{array}\right)\left(1-\frac{k+m}{N}\right)^{n}, \quad k=0,1, \ldots, N-1,
\end{aligned}
$$

описывающую распределение числа пустых ячеек в классической задаче о дробинках, находим, что

$$
\tau_{n k}=\frac{M^{N-k}}{M^{n}} \mathbf{P}\left\{\mu_{0}(n, N)=k\right\}
$$

где $n=1,2, \ldots, k=0,1, \ldots, N-1$. Равенство (2.12) можно написать также, исходя непосредственно из определения вероятностей $\tau_{n k}$. Заметим, что формула (2.12) корректна в том смысле, что выражение в ее правой части не превосходит единицы, так как $\mathbf{P}\left\{\mu_{0}(n, N)=k\right\}>0$ лишь для $k \geqslant N-\min (n, N)$.

Предположив, что условия теоремы выполнены и зафиксировав целое число $k \geqslant 0$, рассмотрим поведение при $N \rightarrow \infty$ вероятности $\mathbf{P}\left\{\widehat{\mu}_{0}(n, N, M)-N+n=k\right\}$, которую согласно (1.1) и (2.12) можно записать следуюшим образом:

$$
\begin{aligned}
\widehat{\tau}_{n, N-n+k} & =\frac{1}{\pi_{n}} \mathbf{P}\left\{\widehat{\mu}_{0}(n, N, M)=N-n+k\right\} \\
& =\frac{1}{\pi_{n}} \frac{\mathbf{P}\left\{\mu_{0}(n, N)-N+n=k\right\}}{M^{k}} \\
n & =1,2, \ldots, \quad k=0,1, \ldots, N-1 .
\end{aligned}
$$

В силу теоремы 3.1 из работы [2] находим, что имеет место сходимость

$$
\lim _{N \rightarrow \infty} \pi_{n}=\exp \left\{-\left(1-\frac{1}{M}\right) \lambda\right\} \text {. }
$$

Учитывая, что при выполнении условий теоремы

$$
\mu_{0}(n, N)-N+n \underset{N \rightarrow \infty}{\Longrightarrow} \Pi(\lambda)
$$

из (2.13) и (2.14) получаем

$$
\lim _{N \rightarrow \infty} \mathbf{P}\left\{\widehat{\mu}_{0}(n, N, M)-N+n=k\right\}=\frac{1}{k !}\left(\frac{\lambda}{M}\right)^{k} e^{-\lambda / M}, \quad k=0,1, \ldots
$$

и, следовательно, (2.11) доказано. 
3. Для доказательства основных результатов работы, которые будут представлены в п. 4, сформулируем и докажем несколько лемм.

Лемма 3.1. Пусть $M \geqslant 2$ фиксированно, а величины $n u N$ изменяются так, что выполнено условие (1.3). Тогда равномерно по $\alpha=n / N, 0<\alpha_{0} \leqslant \alpha \leqslant \alpha_{1}<\infty$, для вероятностей $\pi_{n}$ справедливо асимптотическое представление

$$
\begin{aligned}
\pi_{n}(N, M)= & \left(\frac{M x_{0}}{\alpha}\right)^{(1-\alpha) N} \frac{\exp \left\{\left(x_{0}-\alpha\right) N\right\}}{\sqrt{1+x_{0}-\alpha}} \\
& \times\left(1+\frac{1}{24}\left(\frac{2}{\alpha}+a\right) \frac{1}{N}+O\left(\frac{1}{N^{2}}\right)\right),
\end{aligned}
$$

əde

$$
\begin{aligned}
a= & \frac{3 k_{4}}{\left(1+x_{0}-\alpha\right)^{2}}-\frac{5 k_{3}^{2}}{\left(1+x_{0}-\alpha\right)^{3}} \\
k_{3}= & \alpha(1-\alpha)(1-2 \alpha)+3 \alpha(1-\alpha) x_{0}+\alpha x_{0}^{2}, \\
k_{4}= & \alpha-7 \alpha^{2}+12 \alpha^{3}-6 \alpha^{4}+\alpha\left(7-18 \alpha+12 \alpha^{2}\right) x_{0} \\
& +\alpha(6-7 \alpha) x_{0}^{2}+\alpha x_{0}^{3}
\end{aligned}
$$

и $x_{0}>0$ - единственный корень уравнения

$$
x=\alpha\left(1-\beta e^{-x}\right), \quad \beta=1-\frac{1}{M} .
$$

В формуле (3.1)

$$
1+x_{0}-\alpha>0
$$

$u$

$$
b(\alpha)=\left(\frac{M x_{0}}{\alpha}\right)^{1-\alpha} e^{x_{0}-\alpha}<1 .
$$

Д ок а з а т е л ь с т в о. Согласно (2.2)

$$
1+\sum_{n=1}^{\infty} \pi_{n} \frac{z^{n}}{n !}=\left(1+M\left(e^{z /(N M)}-1\right)\right)^{N}
$$

из (3.6) нахоцим, что

$$
\pi_{n}=\frac{n !}{N^{n} M^{n}} I(n, N)
$$

где

$$
I(n, N)=\frac{1}{2 \pi i} \int_{\mathscr{L}}[A(z)]^{N} \frac{d z}{z^{n+1}},
$$

$A(z)=1+M\left(e^{z}-1\right)$ и $\mathscr{L}$ - простой замкнутый контур, охватываюший точку $z=0$ комплексной плоскости. Асимптотика интегралов вица (3.8) 
в предположении, что $n$ и $N$ удовлетворяют условию (1.3), а функция $A(z)$ разлагается в окрестности точки $z=0$ в степенной ряд с неотрицательными коэффициентами и удовлетворяет некоторым дополнительным условиям, описана в работах [5] (см. также [1]), [6], [7]. В частности, из результатов работы [6] после некоторых вычислений можно вывести следуюшее предложение.

Предложение. Пусть

$$
A(z)=\sum_{n=0}^{\infty} a_{n} z^{n}
$$

- степенной ряд с неотрицательными коэффициентами и радиусом сходимости $R>0$, для которого выполняются следующие условия:

1) $a_{0}>0$ :

2) если $\left\{n_{k}\right\}$ - номера всех ненулевых коэффичиентов $a_{n}$ ряда (3.9), $n_{k} \neq 0$, то числа $n_{k}$ не имеют общего делителя, отличного от единиubl;

3) $\lim _{x \rightarrow R} A(x)=+\infty$.

Тогда, если п и $N$ удовлетворяют условию (1.3), равномерно по $\alpha$, $\alpha=n / N \in\left[\alpha_{0}, \alpha_{1}\right]$, для интеграла (3.8) имеет место асимптотическое разложение

$$
I(n, N)=\frac{\left[A\left(x_{0}\right)\right]^{N}}{x_{0}^{n} \sqrt{2 \pi N k_{2}\left(x_{0}\right)}}\left(1+\frac{a\left(x_{0}\right)}{24 N}+O\left(\frac{1}{N^{2}}\right)\right),
$$

где $x_{0}-$ единственный положительный корень уравнения

$$
x \frac{A^{\prime}(x)}{A(x)}=\alpha
$$

$u$

$$
\begin{aligned}
a\left(x_{0}\right)= & 3 \frac{k_{4}\left(x_{0}\right)}{\left[k_{2}\left(x_{0}\right)\right]^{2}}-5 \frac{k_{3}^{2}\left(x_{0}\right)}{\left[k_{2}\left(x_{0}\right)\right]^{3}} \\
k_{2}\left(x_{0}\right)= & x_{0}^{2} \frac{A^{\prime \prime}\left(x_{0}\right)}{A\left(x_{0}\right)}+\alpha(1-\alpha) \\
k_{3}\left(x_{0}\right)= & 3(1-\alpha) x_{0}^{2} \frac{A^{\prime \prime}\left(x_{0}\right)}{A\left(x_{0}\right)}+x_{0}^{3} \frac{A^{\prime \prime \prime}\left(x_{0}\right)}{A\left(x_{0}\right)}+\alpha(1-\alpha)(1-2 \alpha) \\
k_{4}\left(x_{0}\right)= & \alpha-7 \alpha^{2}+12 \alpha^{3}-6 \alpha^{4}+\left(7-18 \alpha+12 \alpha^{2}\right) x_{0}^{2} \frac{A^{\prime \prime}\left(x_{0}\right)}{A\left(x_{0}\right)} \\
& +\left[2(3-2 \alpha) \frac{A^{\prime \prime \prime}\left(x_{0}\right)}{A\left(x_{0}\right)}+\frac{A^{I V}\left(x_{0}\right)}{A\left(x_{0}\right)}\right] x_{0}^{3}-3 x_{0}^{4} \frac{A^{\prime \prime 2}\left(x_{0}\right)}{A^{2}\left(x_{0}\right)}
\end{aligned}
$$


Используя это предложение с учетом вида нашей функции $A(z)$, указанного выше, с помощью (3.7) убедимся в справедливости разложения (3.1).

Осталось проверить справедливость неравенств (3.4) и (3.5), обеспечиваюших корректность формулы (3.1). Перепишем уравнение (3.3) в виде

$$
\alpha=g(x), \quad g(x)=\frac{x}{1-\beta e^{-x}} .
$$

Имеем:

$$
1+x_{0}-\alpha=\frac{1-\beta\left(1+x_{0}\right) e^{-x_{0}}}{1-\beta e^{-x_{0}}} .
$$

Так как $x_{0}>0, \beta<1$ и $(1+x) e^{-x}<1$ при $x>0$, то правая часть (3.12) положительна и справедливо неравенство (3.4).

Очевидно, $g(0)=0$ и для $x \geqslant 0$

$$
g^{\prime}(x)=\frac{1-\beta(1+x) e^{-x}}{\left(1-\beta e^{-x}\right)^{2}},
$$

так что $g(x)$ монотонно возрастает в промежутке $[0, \infty)$. Следовательно, в этом промежутке $g(x)$ имеет однозначную обратную функцию $x=$ $x(\alpha)$ - корень уравнения (3.11), которая монотонно возрастает при $\alpha \geqslant 0$ и $x(0)=0$. Применяя формулу Бюрмана-Лагранжа, находим, что для корня $x(\alpha)>0$ уравнения (3.11) при $\alpha \rightarrow 0$ имеет место разложение

$$
x(\alpha)=(1-\beta) \alpha+\beta(1-\beta) \alpha^{2}+\frac{1}{2} \beta(1-\beta)(3 \beta-1) \alpha^{3}+O\left(\alpha^{4}\right) .
$$

С его помощью находим, что при $\alpha \rightarrow 0 \quad b(\alpha)=1-\frac{1}{2} \beta \alpha^{2}+O\left(\alpha^{3}\right)$, так что $b(0)=1$ и (3.5) выполняется при малых $\alpha>0$. Далее, для $\alpha>0$, используя (3.13), получаем, что

$$
b^{\prime}(\alpha)=-b(\alpha) \ln \frac{x(\alpha)}{\alpha(1-\beta)} .
$$

Поскольку $x(\alpha)>\alpha(1-\beta)$, то $b^{\prime}(\alpha)<0$ при $\alpha>0$ и, следовательно, (3.5) выполняется для всех $\alpha>0$. Јемма доказана.

Лемма 3.2. В условиях леммы 3.1 равномерно по $\alpha, \alpha=n / N, \alpha=$ $\left[\alpha_{0}, \alpha_{1}\right]$, для математического ожидания и дисперсии сл.в. $\widehat{\mu}_{0}(n, N, M)$ имеют место оченки

$$
\begin{aligned}
& \mathbf{E} \widehat{\mu}_{0}(n, N, M)=N m\left(1+O\left(\frac{1}{N}\right)\right), \\
& \mathbf{D} \widehat{\mu}_{0}(n, N, M)=N b^{2}\left(1+O\left(\frac{1}{N}\right)\right),
\end{aligned}
$$


2de

$$
\begin{aligned}
& m=\frac{\alpha}{M x_{0}} e^{-x_{0}}<1, \\
& b^{2}=m[1-(1+d) m]>0, \quad d=\frac{\alpha}{1+x_{0}-\alpha}
\end{aligned}
$$

и $x_{0}>0-$ корень уравнения (3.3).

3 а м е ч а н и е. В формулировке леммы 3.2 предполагается, что $M \geqslant 2$, но формулы (3.14) и (3.15) верны и при $M=1$. В этом случае уравнение (3.3) имеет тривиальный вид $x=\alpha$, и оценки (3.14) и (3.15) сводятся к известным в классической задаче о дробинках оценкам $\mathbf{E} \mu_{0}(n, N)$ и $\mathbf{D} \mu_{0}(n, N)$ в центральной области [1, с. 13$]$.

При доказательстве предельных теорем, о которых пойдет речь в п. 4, лемма 3.2 непосредственно использоваться не будет. Роль леммы состоит в том, что она раскрывает смысл центрирования и нормирования сл.в. $\widehat{\mu}_{0}$, указанной в этих теоремах.

Доказ а тельство ле м мы 3.2. Согласно (2.7)

$$
\mathbf{E} \widehat{\mu}_{0}(n, N, M)=\frac{N}{\pi_{n}} \cdot \frac{n !}{2 \pi i} \int_{\mathscr{L}}\left(1+M\left(e^{z /(N M)}-1\right)\right)^{N-1} \frac{d z}{z^{n+1}}
$$

где $\mathscr{L}-$ замкнутый контур, охватываюший точку $z=0$. Учитывая оценку $\pi_{n}$, полученную в лемме 3.1 , и используя предложение, сформулированное при доказательстве этой леммы и касающееся асимптотической оценки интегралов вида (3.8), из (3.18) выведем (3.14). При $M=1$ оценка (3.14) есть очевидное следствие первой из формул (2.10).

Более сложным является вывод оценки (3.15). Заметив, что

$$
\mathbf{D} \widehat{\mu}_{0}(n, N, M)=\mathbf{E} \widehat{\mu}_{0}^{[2]}(n, N, M)+\mathbf{E} \widehat{\mu}_{0}(n, N, M)-\left(\mathbf{E} \widehat{\mu}_{0}(n, N, M)\right)^{2},
$$

оценим момент $\mathbf{E} \widehat{\mu}_{0}^{[2]}$, исходя из интегрального представления

$$
\mathbf{E} \widehat{\mu}_{0}^{[2]}(n, N, M)=\frac{N(N-1)}{\pi_{n}} \cdot \frac{n !}{2 \pi i} \int_{\mathscr{L}}\left(1+M\left(e^{z /(N M)}-1\right)\right)^{N-2} \frac{d z}{z^{n+1}}
$$

аналогичного равенству (3.18). Как и в случае оценки $\mathbf{E} \widehat{\mu}_{0}$, нетрудно доказать, что

$$
\mathrm{E} \widehat{\mu}_{0}^{[2]}(n, N, M)=N(N-1) m^{2}\left(1+O\left(\frac{1}{N}\right)\right)
$$

Однако полученных оценок $\mathbf{E} \widehat{\mu}_{0}$ и $\mathbf{E} \widehat{\mu}_{0}^{[2]}$ недостаточно для вывода соотношения (3.15). Для того чтобы доказать (3.15) нужно с помошью представления (3.1) и предложения, связанного с разложением (3.11), найти 
в явном виде коэффициенты $a_{1}$ и $a_{2}$ в разложениях

$$
\begin{aligned}
\mathbf{E} \hat{\mu}_{0} & =N m\left(1+\frac{a_{1}}{N}+O\left(\frac{1}{N^{2}}\right)\right) \\
\mathbf{E} \hat{\mu}_{0}^{[2]} & =N(N-1) m^{2}\left(1+\frac{a_{2}}{N}+O\left(\frac{1}{N^{2}}\right)\right) .
\end{aligned}
$$

Соответствующие вычисления, приводящие в итоге к оценке (3.15), ввиду их громоздкости опущены.

Убедимся в справедливости неравенств, указанных в форму$\operatorname{лах~}(3.16)$ и (3.17). Так как $\alpha(1-\beta)<x_{0}$, то $m=\left(\alpha(1-\beta) / x_{0}\right) e^{-x_{0}}<1$. Для доказательства неравенства $m[1-(1+d) m]>0$, которое оправдывает обозначение $b^{2}$ для его левой части, заметим, что оно выполняется тогда и только тогда, когда

$$
\left(1+\frac{\alpha}{1+x_{0}-\alpha}\right) \frac{\alpha}{M x_{0}} e^{-x_{0}}<1 .
$$

В силу (3.3) последнее неравенство равносильно неравенству

$$
(1-\beta) \frac{\left(1+x_{0}\right) e^{-x_{0}}}{1-\left(1+x_{0}\right) \beta e^{-x_{0}}}<1,
$$

которое имеет место тогда и только тогда, когда $\left(1+x_{0}\right) e^{-x_{0}}<1$. Так как $x_{0}>0$, то отсюда следует, что доказываемое неравенство действительно справедливо. Лемма доказана.

Как было замечено выше, если выполнено условие (1.3), то $\mathbf{E} \mu_{0}(n, N)=N e^{-\alpha}(1+O(1 / N))$. Аналогично доказательству неравенства (3.5) можно показать, что $m<e^{-\alpha}$, где $m$ определяется формулой (3.16) и $M \geqslant 2$. Следовательно, для достаточно больших $N$ будем иметь $\mathbf{E} \mu_{0}(n, N)>\mathbf{E} \widehat{\mu}_{0}(n, N<M), M \geqslant 2$.

Лемма 3.3. Дая условных вероятностей $\widehat{\tau}_{n k}(N, M)$ выполняется соотношение

$$
\widehat{\tau}_{n k}(N, M)=\left(\begin{array}{c}
N \\
k
\end{array}\right)\left(1-\frac{k}{N}\right)^{n} \frac{\tau_{n 0}(N-k, M)}{\pi_{n}(N, M)},
$$

zде $n, N=1,2, \ldots, M=2,3, \ldots, k=1, \ldots, N-1$.

Доказ а те льст в о. Пусть

$$
T_{N M}(z)=T_{N M}(0, z)=\sum_{n=1}^{\infty} \tau_{n 0}(N, M) \frac{z^{n}}{n !}
$$

一 п.ф. вероятностей $\tau_{n 0}(N, M)$. Тогда из (2.6) получаем

$$
T_{N M}(N z)=M^{N}\left(e^{z / M}-1\right)^{N} \text {. }
$$


Учитывая (2.5) и используя (2.6) и (3.20), находим, что

$$
\begin{aligned}
T_{N M}(x, N z) & =x^{N}+\sum_{k=0}^{N-1}\left(\begin{array}{l}
N \\
k
\end{array}\right) x^{k} M^{N-k}\left(e^{z / M}-1\right)^{N-k} \\
& =x^{N}+\sum_{k=0}^{N-1}\left(\begin{array}{l}
N \\
k
\end{array}\right) x^{k} T_{N-k, M}((N-k) z) \\
& =x^{N}+\sum_{n=1}^{\infty} \frac{z^{n}}{n !} \sum_{k=0}^{N-1}\left(\begin{array}{l}
N \\
k
\end{array}\right)(N-k)^{n} \tau_{n 0}(N-k, M) x^{k} .
\end{aligned}
$$

Отсюда и из определения п.ф. $T_{N M}(x, z)$ следует утверждение леммы.

Лемма 3.3 является аналогом следствия 2 из работы [5] (см. также $[1$, c. 50]). Более того, (3.19) справедливо и при $M=1$, так как, полагая в (3.19) $M=1$, получим частный случай формулы (7) из [5].

Лемма 3.4. При $\alpha>0$ корень $x_{0}>0$ уравнения (3.3) является так̆же единственным положительным корнем уравнения

$$
x=\gamma\left(1-e^{-x}\right), \quad \gamma=\frac{\alpha}{1-m}>1,
$$

где т определено посредством (3.16).

Д о к а з а т е л в с т в о. Сначала проверим, что $x_{0}$ - корень уравнения (3.21). Для этого в правую часть (3.21) подставим

$$
\alpha=\frac{x_{0}}{1-\beta e^{-x_{0}}}, \quad m=m(\alpha)=\frac{\alpha(1-\beta)}{x_{0}} e^{-x_{0}}
$$

и получим $x_{0}$, так что $x_{0}$ действительно является корнем уравнения (3.21).

Далее, убедимся, что для любого $\alpha>0$ выполняется неравенство $\gamma>1$. Учитывая формулы (3.22), приходим к выводу, что это неравенство равносильно соотношению $e^{-x_{0}}>1-x_{0}$, справедливому при $x_{0}>0$. Наконец, положив $g_{1}(x)=\gamma\left(1-e^{-x}\right)$, заметим, что $g_{1}(0)=0$, $g_{1}^{\prime}(x)>0$ для $x \geqslant 0$, причем $g_{1}(0)=\gamma>1$ и $\lim _{x \rightarrow \infty} g_{1}(x)=\gamma$. Отсюда вытекает единственность положительного корня уравнения (3.21). Лемма доказана.

Как и лемма 3.3 , лемма 3.4 также справедлива при $M=1$.

4. Перейдем к доказательству локальной предельной теоремы в центральной области изменения $n$ и $N$ для сл.в. $\widehat{\mu}_{0}(n, N, M), M=2,3 \ldots$

Теорема 4.1. Предположим, что выполняется условие (1.3) и $M \geqslant 2$ - фиксированное челое число. Тогда равномерно по $\alpha u k$, $\alpha \in\left[\alpha_{0}, \alpha_{1}\right]$,

$$
k=N m+\sqrt{N} b u, \quad|u| \leqslant C, C>0-\text { константа }
$$


имеет место асимптотическое равенство:

$$
\mathbf{P}\left\{\widehat{\mu}_{0}(n, N, M)=k\right\}=\frac{1}{\sqrt{2 \pi N} b} \exp \left\{-\frac{1}{2} u^{2}\right\}\left(1+O\left(\frac{1}{\sqrt{N}}\right)\right),
$$

aде $m$ и b определнотся формулами (3.16) и (3.17) соответственно.

Д о к а з а т е л ь с т в о. Согласно (1.1) и лемме (3.3)

$$
\mathbf{P}\left\{\widehat{\mu}_{0}(n, N, M)=k\right\}=\frac{N !}{k !(N-k) !} \cdot \frac{(N-k)^{n}}{N^{n}} \cdot \frac{\tau_{n 0}(N-k, M)}{\pi_{n}(N, M)} .
$$

Из равенства (3.20) и определения п.ф. $T_{N M}(z)$, данного при доказательстве леммы 3.3 , находим, что

$$
\tau_{n 0}(N-k, M)=\frac{n ! M^{N-k}}{(N-k)^{n} M^{n}} \cdot \frac{1}{2 \pi i} \int_{\mathscr{L}}\left(e^{z}-1\right)^{N-k} \frac{d z}{z^{n+1}},
$$

где $\mathscr{L}$ - замкнутый контур, охватывающий точку $z=0$ комплексной плоскости. С помощью (3.1) и (4.4) из (4.3) получаем, что

$$
\mathbf{P}\left\{\widehat{\mu}_{0}(n, N, M)=k\right\}=X_{1} X_{2} X_{3} X_{4}\left(1+O\left(\frac{1}{N}\right)\right),
$$

где

$$
\begin{aligned}
& X_{1}=\frac{N !}{k !(N-k) !}, \quad X_{2}=\frac{n !}{N^{n}}, \\
& X_{3}=\frac{1}{M^{k}}\left(\frac{\alpha}{x_{0}}\right)^{(1-\alpha) N} \frac{e^{\left(\alpha-x_{0}\right) N}}{\sqrt{1+x_{0}-\alpha}}, \\
& X_{4}=\frac{1}{2 \pi i} \int_{\mathscr{L}}\left(e^{z}-1\right)^{N-k} \frac{d z}{z^{n+1}} .
\end{aligned}
$$

Нас интересуют асимптотические оценки величин $\ln X_{i}, i=1,2,3,4$. Аналогично соотношению (41) из [5] можно написать оценку

$$
\begin{aligned}
\ln X_{1}= & -[m \ln m+(1-m) \ln (1-m)] N+b u[\ln (1-m)-\ln m] \sqrt{N} \\
& -\ln \sqrt{2 \pi N}-\ln \sqrt{m(1-m)}-\frac{1}{2} u^{2} \frac{b^{2}}{m(1-m)}+O\left(\frac{1}{\sqrt{N}}\right),
\end{aligned}
$$

равномерную по $\alpha, \alpha \in\left[\alpha_{0}, \alpha_{1}\right]$, и $k$ вида (4.1). С помощью формулы Стирлинга, учитывая, что $n=\alpha N$, находим, что равномерно по $\alpha$, $\alpha \in\left[\alpha_{0}, \alpha_{1}\right]$,

$$
X_{2}=(\alpha \ln \alpha-\alpha) N+\ln \sqrt{2 \pi \alpha N}+O\left(\frac{1}{N}\right)
$$


Далее, из (4.6) следует, что

$$
\begin{aligned}
\ln X_{3}= & {\left[m \ln (1-\beta)+\alpha-x_{0}+(1-\alpha) \ln \alpha-(1-\alpha) \ln x_{0}\right] N } \\
& +\ln (1-\beta) \cdot b u \sqrt{N}+\ln \sqrt{1+x_{0}-\alpha}
\end{aligned}
$$

Приступая к оцениванию $\ln X_{4}$, определим в правой полуплоскости функцию

$$
f(z)=\ln A(z)-\alpha_{k} \ln z ; \quad \alpha_{k}=\frac{n}{N-k}, A(z)=e^{z}-1
$$

принимающую действительные значения для действительных $z$. Учитывая (4.1), находим, что

$$
\alpha_{k}=a_{0}+\frac{a_{1}}{\sqrt{N}}+\frac{a_{2}}{N}+O\left(\frac{1}{N \sqrt{N}}\right)
$$

где

$$
a_{0}=\frac{\alpha}{1-m}, \quad a_{1}=\frac{\alpha b}{(1-m)^{2}} u, \quad a_{2}=\frac{\alpha b^{2}}{(1-m)^{3}} u^{2}
$$

Рассмотрим для $x>0$ уравнение $f^{\prime}(x)=0$; согласно определению $A(z)$ и $f(z)$ оно приводится к виду

$$
x=\alpha_{k}\left(1-e^{-x}\right) .
$$

В силу (4.12) и утверждения леммы 3.4 относительно величины $\alpha /(1-m)$ найдутся такие $\varepsilon>0$ и $N_{0} \geqslant 1$, что для всех $\alpha \in\left[\alpha^{(0)}, \alpha^{(1)}\right]$, и $k$ вида (4.1) при $N \geqslant N_{0}$ будут справедливы неравенства

$$
1<\frac{\alpha^{(0)}}{1-m}-\varepsilon \leqslant \alpha_{k} \leqslant \frac{\alpha^{(1)}}{1-m}+\varepsilon<\infty .
$$

Из (4.15) и из леммы 2 работы [5] вытекает, что при $N \geqslant N_{0}$ для указанных значений $\alpha$ и $k$ уравнение (4.14) будет иметь единственное решение $x_{k}>0$.

Проверим, что для тех же $N, \alpha$ и $k$ выполняется соотношение

$$
f^{\prime \prime}\left(x_{k}\right) \geqslant \delta>0 \text {. }
$$

Во-первых, из (4.11) с учетом того, что $x_{k}$ - корень уравнения (4.14), следует, что

$$
f^{\prime \prime}\left(x_{k}\right)=\left(1+x_{k}-\alpha_{k}\right) \frac{\alpha_{k}}{x_{k}^{2}}
$$

Затем найдем первые члены разложения $x_{k}$ по степеням $1 / \sqrt{N}$. Полагая

$$
x_{k}=s_{0}+\frac{s_{1}}{\sqrt{N}}+\frac{s_{2}}{N}+O\left(\frac{1}{N \sqrt{N}}\right)
$$


и используя (4.12), из (4.14) для величин $s_{0}$ и $s_{1}$, получим уравнения:

$$
\begin{aligned}
& s_{0}=a_{0}\left(1-e^{-s_{0}}\right), \\
& s_{1}=a_{0} s_{1} e^{-s_{0}}+a_{1}\left(1-e^{-s_{0}}\right) .
\end{aligned}
$$

Из (4.13) и леммы 3.4 заключаем, что

$$
s_{0}=x_{0}
$$

где $x_{0}$ - единственный положительный корень уравнения (3.3). Ввиду (4.21) и формул (4.13), определяющих $a_{0}$ и $a_{1}$, из (4.20) вытекает, что

$$
s_{1}=\frac{x_{0} b}{(1-m) H} u, \quad H=1+x_{0}-\frac{\alpha}{1-m} .
$$

Явное выражение для коэффициента $s_{2}$ из разложения (4.18), как выяснится в дальнейшем, нам не потребуется (см. (4.29) ниже). С помошью (4.12), (4.13), (4.18) и (4.21) находим, что

$$
1+x_{k}-\alpha_{k}=H+O\left(\frac{1}{\sqrt{N}}\right) \text {. }
$$

Из неравенств (3.4) и (3.17) несложно вывести, что $H>0$. Отсюда и из (4.17) и (4.23) следует, что при достаточно больших $N \geqslant N_{0}^{\prime}$ для всех $\alpha \in\left[\alpha^{(0)}, \alpha^{(1)}\right]$ и $k$ вида (4.1) выполняется неравенство (4.16).

Следовательно, можно воспользоваться теоремой 3 из [5] для асимптотической оценки интеграла (4.7). В результате убедимся, что равномерно по $\alpha, \alpha \in\left[\alpha^{(0)}, \alpha^{(1)}\right]$, и $k$ вида (4.1) для достаточно больших $N \geqslant \max \left(N_{0}, N_{0}^{\prime}\right)$

$$
X_{4}=\frac{1}{\sqrt{2 \pi \alpha N}} \cdot \frac{\left(e^{x_{k}}-1\right)^{N-k}}{x_{k}^{\alpha N} \sqrt{1+x_{k}-\alpha_{k}}} \cdot\left(1+O\left(\frac{1}{N}\right)\right),
$$

вследствие чего, учитывая (4.23), получим

$$
\ln X_{4}=\ln \left[\left(e^{x_{k}}-1\right)^{N-k} x_{k}^{-\alpha N}\right]-\ln \sqrt{2 \pi \alpha N}-\ln \sqrt{H}+O\left(\frac{1}{N}\right) .
$$

С помощью (4.18) нетрудно убедиться, что

$$
\ln \left(e^{x_{k}}-1\right)=A_{0}+\frac{A_{1}}{\sqrt{N}}+\frac{A_{2}}{N}+O\left(\frac{1}{N \sqrt{N}}\right)
$$

где

$$
\begin{aligned}
& A_{0}=x_{0}+\ln x_{0}+\ln \frac{1-m}{\alpha}, \quad A_{1}=\frac{\alpha}{(1-m) x_{0}} s_{1}, \\
& A_{2}=\frac{\alpha}{(1-m) x_{0}}\left(s_{2}+\frac{1}{2} s_{1}^{2}\right)-\frac{1}{2} \frac{\alpha^{2}}{(1-m)^{2} x_{0}^{2}} s_{1}^{2} .
\end{aligned}
$$


Из (4.24), (4.25) и (4.1) следует, что

$$
\begin{aligned}
\ln \left(e^{x_{k}}-1\right)^{N-k}= & (1-m) A_{0} N+\left[(1-m) A_{1}+b u A_{0}\right] \sqrt{N} \\
& +(1-m) A_{2}-b u A_{1}+O\left(\frac{1}{\sqrt{N}}\right)
\end{aligned}
$$

где $A_{0}, A_{1}$ и $A_{2}$ определяются формулами (4.26).

Далее, снова используя (4.18), напишем разложение для $\ln \left(x_{k}^{-\alpha N}\right)$ :

$$
\ln \left(x_{k}^{-\alpha N}\right)=-\alpha N \ln x_{0}-\frac{\alpha}{x_{0}} s_{1} \sqrt{N}-\frac{\alpha}{x_{0}}\left(s_{2}-\frac{s_{1}^{2}}{2 x_{0}}\right)+O\left(\frac{1}{\sqrt{N}}\right) .
$$

Из (4.27) и (4.28), приняв во внимание формулы (4.26), находим, что

$$
\begin{aligned}
\ln [ & \left.\left(e^{x_{k}}-1\right)^{N-k} x_{k}^{-\alpha N}\right] \\
= & {\left[(1-m)\left(x_{0}+\ln x_{0}+\ln (1-m)-\ln \alpha\right)-\alpha \ln x_{0}\right] N } \\
& -b u\left[x_{0}+\ln x_{0}+\ln (1-m)-\ln \alpha\right] \sqrt{N}+\frac{\alpha}{2 x_{0}} s_{1}^{2} \\
& -\frac{1}{2} \frac{\alpha^{2} s_{1}^{2}}{(1-m) x_{0}^{2}}+\frac{\alpha s_{1}^{2}}{2 x_{0}^{2}}-\frac{\alpha}{(1-m) x_{0}} b u s_{1}+O\left(\frac{1}{\sqrt{N}}\right) .
\end{aligned}
$$

Теперь у нас имеется все необходимое, чтобы завернить доказательство теоремы. Суммируя с учетом (4.29) правые части равенств (4.8), $(4.9),(4.10)$ и $(4.24)$, из (4.5) получаем, что

$$
\ln \mathbf{P}\left\{\widehat{\mu}_{0}(n, N, M)=k\right\}=-\ln \sqrt{2 \pi N}+\ln \sqrt{\frac{1+x_{0}-\alpha}{m(1-m) H}}+\sigma .
$$

Здесь

$\sigma=\alpha\left(\frac{1}{2 x_{0}}-\frac{\alpha}{2(1-m) x_{0}^{2}}+\frac{1}{2 x_{0}^{2}}\right) s_{1}^{2}-\frac{\alpha}{(1-m) x_{0}} b u s_{1}-\frac{1}{2} u^{2} \frac{b^{2}}{m(1-m)}$.

Учитывая определения величин $b$ и $H$, а также выражение для $s_{1}$ (см. (3.17) и (4.22)), нетрудно проверить, что

$$
H=\frac{1+x_{0}-\alpha}{m(1-m)} b^{2}
$$

а формула для $\sigma$ упрощается и в конечном итоге приводится к виду

$$
\sigma=-\frac{u^{2}}{2}
$$

Из (4.30), (4.31) и (4.32) вытекает справедливость равенства (4.2). Теорема доказана. 
Доказательство теоремы 4.1 было проведено по схеме, использованной в [1] и [5]. Ключевую роль в этом играет равенство (3.19).

В заключение приведем формулировку интегральной теоремы.

Теорема 4.2. Если выполнено условие (1.3) и $M \geqslant 2-$ биксированное челое число, то имеет место сходимость

$$
\frac{\widehat{\mu}_{0}(n, N, M)-N m}{\sqrt{N} b} \underset{N \rightarrow \infty}{\Longrightarrow} \mathcal{N}(0,1),
$$

аде $\mathscr{N}(0,1)$ - сл.6., распределенная по нормальному закону с параметрами $(0,1)$.

Доказательство теоремы 4.2 можно получить обычным путем из локальной теоремы.

\section{СПИСОК ЛИТЕРАТУРЫ}

1. Колчин В. Ф., Севастьяяов Б. А., Чистяков В. ПІ. Случайные размещения. М.: Наука, 1976, 223 c.

2. Селиванов Б. И. О времени ожидания в схеме случайных размешцений окралшенных частиц. - Дискрет. матем., 1995, т. 7, в. 1, с. 134-144.

3. Békessy A. On classical occupancy problem, I. - A Maguar Tudom. Akad. Matem. Kutató Intéz. Közl., 1963, ser. A, v. 8, № 1-2, p. 59-70.

4. Rényi A. Three new proofs and a generalization of a theorem of Irving Weiss. A Maguar Tudom. Akad. Matem. Kutató Intéz. Közl., 1962, ser. A, v. 7, № 1-2, p. 203-213.

5. Севастьянов Б. А., Чистяков В. П. Асимптотическая нормальность в классической задаче о дробинках. - Теория вероятн. и ее примен., 1964, т. 9, в. 2, c. $223-237$.

6. Good I. J. Saddle-points methods for the multinomial distribution. - Ann. Math. Statist., 1957, v. 28 , № 4, p. 861-881.

7. Федорюх М. В. Асимптотика: Интегралы и ряды. М.: Наука, 1987, 544 с.

Поступила в редакцию 